\title{
Control of magnetically suspended rotor combined with motor drive system *
}

\author{
Gert L. Kruger* George van Schoor* Pieter A. van Vuuren* \\ * North-West University, Potchefstroom, 11 Hoffman Street (Tel: 018 \\ 299 1904; e-mail: krugergl@gmail.com, George.VanSchoor@nwu.ac.za, \\ Pieter.VanVuuren@nwu.ac.za).
}

\begin{abstract}
This paper presents the cooperative control between the active magnetic bearing system and the permanent magnet synchronous drive. More specifically, the coupling between the vibration control and sensorless drive control is considered since both systems have the ability to estimate the angular speed. The coupling of the estimated speed in each controller is considered. Also, a modified start-up procedure for the sensorless vector control had to be developed when the drive is combined with the AMB system to accommodate the low friction which does not yield damping of the speed perturbation at start-up. Simulation results are presented for the developed control using detailed models for both systems.
\end{abstract}

Keywords:

Vibration control; Magnetic Bearings; Sensorless control

\section{INTRODUCTION}

An active magnetic bearing is an example of a mechatronic system [Worlitz, 2011]. Its purpose is to suspend an object, typically a rotor, such that it can rotate freely. The active magnetic bearing (AMB) achieves this objective by incorporating components that originate from diverse engineering disciplines. As shown in Fig. 1, the force required to suspend a rotor is generated by electromagnets. The required currents in the electromagnets are computed in a controller which take a rotor position measurement as input. The current references generated by the control software are actuated by power amplifiers (usually IGBT based). The control software needs to continuously update the reference current since the AMB system is inherently unstable [Schweitzer et al., 2009]. Usually, there is a normal roller element bearing with an inner diameter larger than the rotor, to catch the rotor in case of $\mathrm{AMB}$ failure.

The AMB has many advantages over conventional roller element bearings due to its rotation without contact, such as lower frictional losses and wear, environmentally friendly, lubrication free operation and fault detection capability [Sawicki et al., 2008, Lees et al., 2009, Gouws and van Schoor, 2009]. The advantages of the AMB make it preferred in applications such as machine tool spindles [Bleuler et al., 1994], vacuum systems, turbo-machinery, fly-wheel energy storage [Polajžer, 2010] and inertial wheel systems used for attitude control of satellites Gerlach et al. [2006], Alain and Lechable [1996]. AMBs are also considered for jet engines Burdet [2006].

Another advantage of the AMB system is that it has the ability to control vibration. Vibration control has either one of two possible goals, either to reduce the rotor vibra-

* This work was supported in part by the National Research Foundation under Grant UID: 82117.

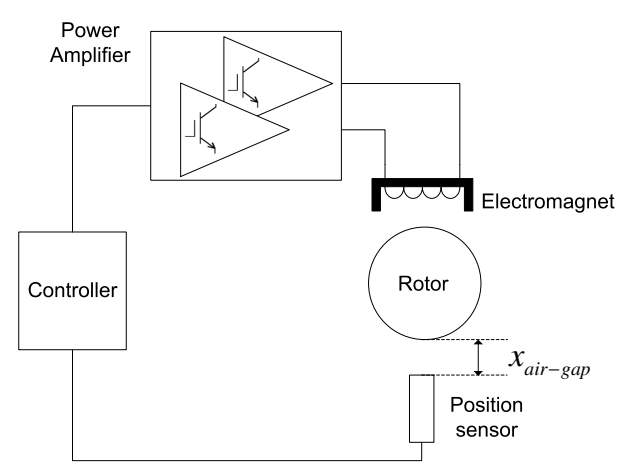

Fig. 1. Active magnetic bearing system.

tion (i.e. rotate around the geometric centre) or to allow the rotor to vibrate and thereby prevent the synchronous force from being generated by the AMB (i.e. rotate around the inertial axis). Control about the geometric centre is useful in applications where high precision is required such as machine tool spindles Grochmal and Lynch [2007]. Rotation about the inertial axis is useful in applications where the vibration transmitted to the machine housing need to be minimized. The unbalance can be modelled as a measurement error as shown in Fig. 2. Sensors feed back the measured geometric centre which differs from the inertial centre.

Several control methods have been proposed to achieve these vibration control aims. These techniques can be broadly classified into open-and closed-loop control techniques. The open-loop control techniques have a priori information on the unbalance, so that a synchronous signal can be generated with only the rotor angular position as input. The closed-loop techniques use the position feedback and injects a synchronous signal in order to cancel synchronous disturbances. The stability of the closedloop methods need to be considered during design. Some 
methods can be classified as semi-closed-loop, since they determine the unbalance parameters on-line, after which their input can be switched off, but with the synchronous compensation signal still being generated. Early vibration control methods introduced a speed dependent notch filter in order to cancel the synchronous measurement disturbance. Herzog et al. [1996] presented a method of designing a multiple-input multiple-output notch filter using the sensitivity function in order to attain a stable control. Kejian et al. [2012] have shown that the unbalance compensation can be designed in such a manner so that the same unbalance compensation can be used for both a geometric centre and an inertial axis control, simply by changing the pickup and insertion points with respect to the stabilization control loop.

In the case of inertial axis control the AMB may be designed with a smaller dynamic force range, with a resulting smaller power amplifier rating. The power amplifier is also less likely to saturate when combined with this control. In the case of inertial axis control, the AMB experiences a varying air-gap, which due to the AMB negative stiffness would cause a resulting force which still need to be compensated by the inertial axis control. The inertial axis control compensates the force due to the negative stiffness with a resulting synchronous current. The net synchronous force in this case is zero. Besides the inertial axis and geometric centre control, there is another possibility for vibration control, namely the zero current control as presented by Lee et al. [2002]. Instead of minimizing the reaction force of the $\mathrm{AMB}$, the AMB control current is minimized. This synchronous current may (though unlikely) still saturate the AMB power amplifier. The zero current control does not compensate the synchronous force due to negative stiffness, which is small compared to the mass imbalance force.

All the vibration control techniques require an accurate speed signal that is synchronized with the rotor. The unbalance compensation proposed by Lee et al. [2002] also estimated the speed signal information from the unbalance compensation signal. They also note (without showing) that the speed signal estimated from a sensorless drive control technique can be used as the speed signal input for the unbalance control. The effectiveness of using an estimated speed signal from the sensorless drive have not been tested. Lee et al. also used the speed signal from the unbalance compensation as the feedback in a low dynamic induction machine drive and suggested that it be tested on a vector controlled drive.

The combination of the AMB unbalance control and the sensorless PMSM drive is the focus of this paper. It is tested on the complete integrated simulation model of an AMB and PMSM model with parameters from an actual flywheel energy storage system. Some AMB systems, often found in control research laboratories, have an air turbine for propulsion, but most AMB systems used in the industry have an electrical drive system. These systems usually work independently and the full cooperative control between the AMB and the drive has not yet been fully exploited. It has been suggested by Lima et al. [2010] that the estimated drive speed signal be used as a back-up for the speed signal used in the AMB system and goes forth to present the estimation of the speed using a neuro-fuzzy speed estimator, but does not show the AMB response to the estimated speed signal. By removing the speed sensor and/or using the sensorless drive speed estimation in the unbalance control the reliability of the system increases and complements the efforts of the self-sensing AMBs. By combining these systems the communication between the drive and AMB becomes a point of failure. Jastrzebski et al. [2006] implemented the control of ten power amplifiers for a 5 DOF AMB on a single FPGA. The next logical step is to include the sensorless drive control on the same FPGA, thereby reducing communication bottle-neck and chances of communication failure.

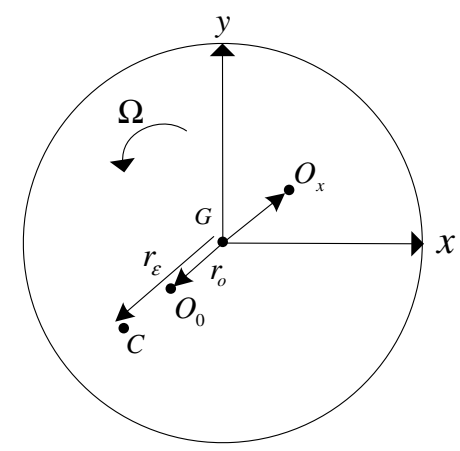

Fig. 2. Rotor unbalance modelled as a measurement error.

In the following sections the models for the AMB and PMSM are presented, followed by the control design of each of these systems, after which simulation results are presented for the cooperative control of these systems.

\section{MATHEMATICAL MODELS}

\subsection{AMB model}

The model of the AMB can be divided into two parts: the equations describing the electromagnetic force and the set of equations describing the rotor. The usual force equation for a single AMB actuator presented in the literature (e.g. Schweitzer et al. [2009]), is:

$$
F=K\left[\left(\frac{i_{0}+i_{c}}{x_{0}-x}\right)^{2}\right]
$$

where $K=K=\frac{1}{4} \mu_{0} N^{2} A_{g} \cos (\theta), A_{g}$ is the pole area and $N$ is the number of winding turns. Clearly, the generated force is non-linearly related to the control current $\left(i_{c}\right)$ and rotor position $(x)$ within the nominal air-gap $\left(x_{0}\right)$. The $\cos (\theta)$ term derives from the angle at which the two poles of a horse-shoe electromagnet acts upon the rotor.

Since a single AMB actuator can actuate force only in a single direction, two actuators are placed in an opposing differential pair. The bias current, $i_{0}$, serves to linearise the force-current relationship to a great extent.

The model of the rotor used in this study assumes a rigid rotor model, as presented by Dimond et al. [2012]. The rotor's equation of motion supported by AMBs is:

$$
\boldsymbol{M} \ddot{\boldsymbol{u}}_{g}+\Omega \boldsymbol{G} \dot{\boldsymbol{u}}_{g}=\boldsymbol{f}_{g, A M B}
$$

where $\Omega$ is the angular frequency of the rotor, $\boldsymbol{M}$ is a diagonal inertia matrix given by: 


$$
\boldsymbol{M}=\left[\begin{array}{cc}
M \boldsymbol{I}_{2 \times 2} & \mathbf{0}_{2 \times 2} \\
\mathbf{0}_{2 \times 2} & J_{t} \boldsymbol{I}_{2 \times 2}
\end{array}\right]
$$

and $\boldsymbol{G}$ is the gyroscopic matrix given by:

$$
\boldsymbol{G}=J_{p}\left[\begin{array}{ccc}
\mathbf{0}_{2 \times 2} & \mathbf{0}_{2 \times 2} \\
\mathbf{0}_{2 \times 2} & 0 & 1 \\
& -1 & 0
\end{array}\right]
$$

$M$ is the mass of the rotor, $J_{t}$ and $J_{p}$ are the transverse and polar moments of inertia (MOI), respectively. The vector $\boldsymbol{u}_{\boldsymbol{g}}=\left[\begin{array}{llll}x_{g} & y_{g} & \theta_{x g} & \theta_{y g}\end{array}\right]$, gives the orientation of the rotor in the centre of gravity coordinates (COG). The input to the rotor in COG coordinates is $\boldsymbol{f}_{g, A M B}=$ $\left[\begin{array}{llll}F_{x g} & F_{y g} & M_{x g} & M_{y g}\end{array}\right]$. In order to use this model, the input force generated by the AMB model at the bearing coordinates need to be transformed to the COG coordinates and the resulting output need to be transformed to the sensor coordinates. These transformations are given by Dimond et al. [2012]:

$$
\begin{gathered}
\underbrace{\left[\begin{array}{c}
F_{x g} \\
F_{y g} \\
M_{x g} \\
M_{y g}
\end{array}\right]}_{\boldsymbol{f}_{g, A M B}}=\underbrace{\left[\begin{array}{cccc}
1 & 0 & 1 & 0 \\
0 & 1 & 0 & 1 \\
0 & a & 0 & -b \\
-a & 0 & b & 0
\end{array}\right]}_{\boldsymbol{Q}_{b}^{T}} \underbrace{\left[\begin{array}{l}
F_{x 1 b} \\
F_{y 1 b} \\
F_{x 2 b} \\
F_{y 2 b}
\end{array}\right]}_{\boldsymbol{f}_{b, A M B}} \\
\underbrace{\left[\begin{array}{l}
x_{1 s} \\
y_{1 s} \\
x_{2 s} \\
y_{2 s}
\end{array}\right]}_{\boldsymbol{u}_{s}}=\underbrace{\left[\begin{array}{cccc}
1 & 0 & 0 & -c \\
0 & 1 & c & 0 \\
1 & 0 & 0 & d \\
0 & 1 & -d & 0
\end{array}\right]}_{\boldsymbol{Q}_{s}} \underbrace{\left[\begin{array}{c}
x_{g} \\
y_{g} \\
\theta_{x g} \\
\theta_{y g}
\end{array}\right]}_{\boldsymbol{u}_{g}}
\end{gathered}
$$

Finally, equation (2) needs to be transformed from second order to first order for numerical implementation. This is depicted in Fig. 3. A static unbalance model is used in the $\mathrm{AMB}$, by adding the eccentricity vector, $r_{\epsilon}=$ $\left|r_{\epsilon}\right|[\sin (\Omega t) \cos (\Omega t)]^{T}$, to the geometric centre in the COG coordinates before it is transformed to the sensor coordinates.

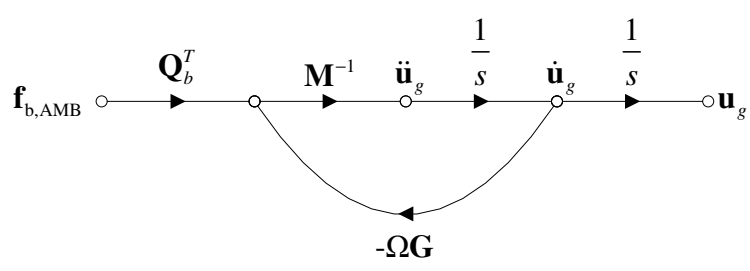

Fig. 3. Signal flow diagram of the rigid rotor plant model.

The AMB and rigid rotor model parameters are presented in Table A.1.

\subsection{Drive model}

The model of the PMSM used in the simulations are the differential equations of the PMSM transformed to the rotor reference frame (adapted from [Perera et al., 2003]):

$$
\begin{aligned}
p i_{d} & =-\frac{1}{\tau_{s}} i_{d}+\omega_{r} \sigma i_{q}+\frac{v_{d}}{L_{d}} \\
p i_{q} & =-\frac{\omega_{r}}{\sigma}\left(\frac{\lambda_{p}}{L_{d}}+i_{d}\right)-\frac{1}{\sigma \tau_{s}} i_{q}+\frac{v_{q}}{L_{q}} \\
p \omega_{m} & =\frac{1}{J_{p}}\left(T_{e}-B \omega_{m}-T_{l}\right) \\
T_{e} & =\frac{3}{2} z_{p}\left(\lambda_{p} i_{q}+L_{d}(1-\sigma) i_{q} i_{d}\right)
\end{aligned}
$$

where $p$ is the time differential operator, $z_{p}$ is the number of pole pairs, $\sigma=\frac{L_{q}}{L_{d}}$ is the saliency factor, $\tau_{s}=\frac{L_{d}}{r_{s}}$ is the d-axis stator electrical time constant, $\lambda_{p}$ is the peak flux linkage due to the permanent magnet and $B$ is the viscous friction coefficient. The inputs to the model is the transformed terminal voltage $\boldsymbol{v}_{d q}$ and load torque $T_{l}$. The state variables of the model is the transformed stator currents $\boldsymbol{i}_{d q}$ and the rotor angular frequency $\omega_{m}\left(\omega_{r}=z_{p} \omega_{m}\right.$ is the angular frequency in electrical coordinates). The PMSM used in this study has a surface mounted permanent magnet, for which the saliency factor is assumed unity. The mechanical angular frequency of the PMSM is coupled to that of the AMB model. In certain simulations it is sufficient to couple a previous PMSM speed response simulation to the AMB, whilst in others, where the PMSM control is dependent on the AMB unbalance control response, both models have to be coupled in the same simulation.

An average value inverter model is used in the simulation in order to reduce the computation time, since the model is already complex due to the coupling between the PMSM and AMB models.

The PMSM model parameters are presented in Table A.2.

\section{AMB CONTROL DESIGN}

A decentralized PD control is used for the AMB. The PD control values as designed by Myburgh [2007] is used. Only the radial AMBs are considered since they are assumed decoupled from the axial AMB. The unbalance control design is performed by following the procedure outlined by Lee et al. [2002]. Note that an integrator gain may be used, but has to be small since the unbalance control derivation assumed only PD control. Fig. (4) depicts the unbalance compensation, $N$, where $G_{c}$ is the PD controller and $G_{p}$ represents the plant model in COG coordinates. The estimated speed is obtained from the unbalance compensation via numerical differentiation of the compensation signal's angle, i.e. $\hat{\omega}_{U B C}=\frac{d}{d t} \arctan \left(\frac{r_{s o_{-} 1 y}}{r_{s o_{-} 1 x}}\right)$. As suggested by Lee et al. [2002] a low pass filter is also used to reduce the noise of the estimated speed. Note that only a single plane's compensation is depicted. A similar loop, but with parameters unique to that $\mathrm{AMB}$ is used for the other AMB. The estimated speed of the AMB plane with the larger inertia was used. Further investigation is required to determine the optimal combination of both planes' estimated speed. The designed AMB control parameters are presented in Table 1.

\section{SENSORLESS PMSM CONTROL DESIGN}

The sensorless vector control design procedure as presented by [Kshirsagar et al., 2006] was followed. The con- 


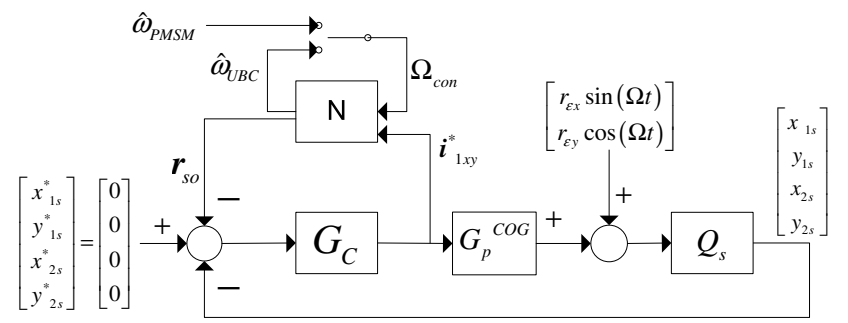

Fig. 4. Unbalance compensation control block diagram.

Table 1. AMB control parameters

\begin{tabular}{|l|c|c|}
\hline Parameter description & Symbol & Value \\
\hline \hline Bottom AMB's PD gain & $K_{p 1}, K_{d 1}$ & $14.2 \times 10^{3}, 30$ \\
\hline Top AMB's PD gain & $K_{p 2}, K_{d 2}$ & $14.2 \times 10^{3}, 30$ \\
\hline $\begin{array}{l}\text { Unbalance control convergence } \\
\text { factor }\end{array}$ & $\tau$ & $50 \times 10^{3}$ \\
\hline Speed estimator's low pass filter & $T_{\hat{\omega} L P F}$ & $\frac{2 \pi 10}{s+2 \pi 10}$ \\
\hline
\end{tabular}

troller consists out of a state observer, which estimates the electrical variables in the estimated coordinate reference frame, $\hat{d} q$. The observer in the rotating reference frame makes use of decoupling in order to partially linearise the state matrix. This has the advantage that less terms in the observer gain matrix need to be gain scheduled as compared to the observer in stationary coordinates as presented by Batzel [2000]. A PLL-based tracking controller receives the estimated back-emf in the estimated $\hat{d}$-axis, $e_{d}^{\hat{r}}$, as input. If the estimated and actual rotor reference frames do not coincide, then $e_{d}^{\hat{r}}$ is such that a PI controller accumulates this error. The output of the tracking controller is the estimated angular velocity, $\hat{\omega}_{r}$, which is integrated to yield the estimated rotor angle, $\hat{\theta}_{r}$. The estimated angle is used to transform the measured current and voltage to the estimated reference frame. Since the angular speed is effectively the derivative of the angular position, this sensorless control scheme is robust against a change in the permanent magnet flux (e.g. due to temperature dependence).

Other sensorless control schemes presented in the literature often derive the required estimated variables in a two step approach. One observer for the estimated position and another for the speed, such as the sensorless control approach as presented by Kim and Sul [2000]. The estimated speed drift is compensated by differentiating the estimated angular position over a longer period. The complexity of this sensorless controller is much less than the one used in this paper. Also, it stands to benefit by compensating the estimated angular speed from the AMBs unbalance compensation, instead of using the estimated speed obtained by differentiation, which has its own drawbacks such as instability at low speed due to the low signal-to-noise ratio of the estimated angular position. Also it is possible to increase the speed estimation accuracy of the unbalance compensation algorithm, by artificially increasing the rotor unbalance at low speed by specifying a non-zero current reference for the unbalance compensation scheme (point $O_{x}$ in Fig. (2)).

The designed sensorless control parameters used in this study are presented in Table (2). Note that both the AMB and PMSM controllers use a sampling period of $100 \mu \mathrm{s}$.
Table 2. Sensorless control parameters.

\begin{tabular}{|l|c|c|}
\hline Parameter description & Symbol & Value \\
\hline \hline Current control PI gain & $K_{p_{-} c}, K_{i_{-} c}$ & $0.5,172$ \\
\hline Speed control PI gain & $K_{p_{-} s}, K_{i_{-}}$ & $0.65,12$ \\
\hline Tracking controller PI gain & $K_{p_{-} t}, K_{i_{-} t}$ & $177,1.58 \times 10^{4}$ \\
\hline Switch-over frequency & $f_{r_{-} i n i t}$ & $15 \mathrm{~Hz}$ \\
\hline State observer bandwidth & $f_{o}$ & $200 \mathrm{~Hz}$ \\
\hline Current limit & $i_{\text {max }}$ & $25 \mathrm{~A}$ \\
\hline DC bus voltage & $V_{\text {bus }}$ & $350 \mathrm{~V}$ \\
\hline
\end{tabular}

\subsection{Modified start-up procedure}

The sensorless vector control used requires an alignment step, followed by an open-loop start-up. After the speed and position observers have settled, the control can switch over to the closed-loop vector control. The open-loop control suggested by [Kshirsagar et al., 2006] uses a constant current vector reference rotating at an angular speed equal to the reference frequency. It has been found that the extremely low friction due to the AMB system, results in little angular speed perturbation damping during the open-loop start-up. This problem is remedied by reducing the current control loop gain by a factor of a hundred during the open-loop start-up. Note that the inverter nonideality, such as dead-time, already decreases the effective current control loop gain Kruger et al. [2013]. Hence the reduced current control gain will have to be tuned on the actual system in order to yield best results.

It has also been found that the current and speed control loop integrators need to be reset on the switch-over instant, to what their outputs would have been during openloop control if cast to the estimated reference frame. This bumpless transfer is especially important for low inertia rotors which may lose synchronization due to the transient that results due to improper transfer from open-to closedloop control. The speed control's integrator is reset to

$$
K_{i_{-} s}^{o}\left(n_{s o}\right)=\hat{i}_{q}^{\hat{r}}\left(n_{s o}-1\right)-K_{p_{-} s} \tilde{\omega}\left(n_{s o}\right)
$$

where $\tilde{\omega}$ is the speed control error, $\hat{i}_{q}^{\hat{r}}$ is the current estimated by the observer and $n_{\text {so }}$ is the switch-over control cycle. The current control loop's integrator is reset to:

$$
K_{i_{-} c}^{o}\left(n_{s o}\right)=v_{d q}^{\hat{r} *}\left(n_{s o}-1\right)-v_{d q_{-} D N}\left(n_{s o}\right)-K_{p_{-} c} \tilde{i}_{d q}^{\hat{r}}\left(n_{s o}\right)
$$

where $v_{d q}^{\hat{r}^{*}}$ is the reference voltage of the previous control cycle transformed to the estimated rotor reference frame, $v_{d q_{-} D N}$ is the feed-forward decoupling term and $\tilde{i}_{d q}^{\hat{r}}$ is the current control error in the estimated reference frame for the current control cycle. The $n_{s o}$ dependence of the terms on both sides of equations (11) and (12) imply that the control algorithm need be calculated in a certain order such that the terms on the right hand sides which depend on $n_{\text {so }}$ have already been evaluated by the time that these equations are processed.

\section{SIMULATION RESULTS}

The simulation results presented in this section highlights the control issues regarding the cooperative control between the AMB and PMSM drive systems. The simulation is performed in Simulink ${ }^{\circledR}$. Band limited white noise representative to that which has been observed in the actual 


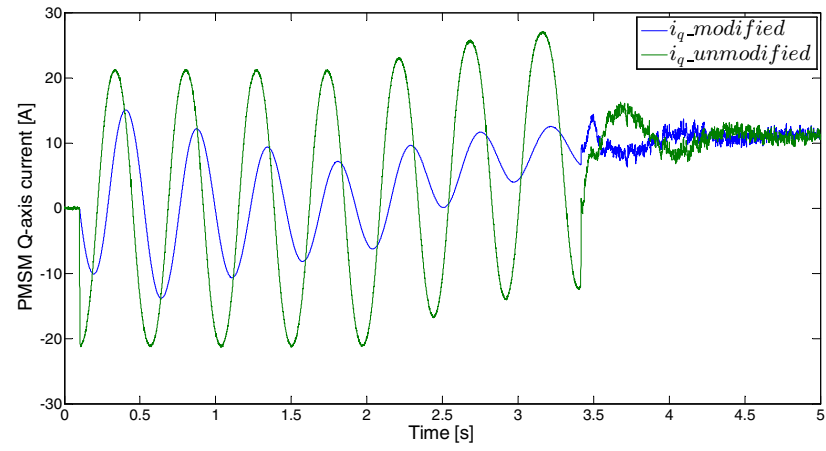

Fig. 5. Modified start-up procedure for the combined AMB and PMSM system.

system is added to all relevant input signals. In order to make the simulation run time faster and simulation results more illustrative the rotor polar moment of inertia used in simulation is reduced by a factor of ten, i.e. $J_{p}=1.07 \times$ $10^{-2} \mathrm{~kg} \cdot \mathrm{m}^{2}$.

Fig. (5) depicts the response with and without the modified start-up procedure (which includes the integrator initialization upon control switch-over). As can be seen the current oscillation with the modified start-up procedure have decayed and has a much smaller current disturbance when the vector control is switched from open-to closedloop.

The estimated speed of the unbalance compensation, with the drive's estimated speed as input, for a rotor runup from standstill to $83 \mathrm{~Hz}$ is depicted in Fig. (6). The experiment repeated, but with the unbalance control's estimated speed as input is also depicted in the other trace of the same figure. At $t=10 \mathrm{~s}$ a torque load of $1 \mathrm{~N} . \mathrm{m}$ is applied. As can be seen, the speed estimation error is very small for both cases. The previous experiment is repeated but with the speed estimator's low pass filter cutoff frequency increased to $15 \mathrm{~Hz}$, in which case a critical frequency is excited. Hence, the speed estimation low pass filter, which was previously used to only reduce noise also determines the stability of the unbalance controller.

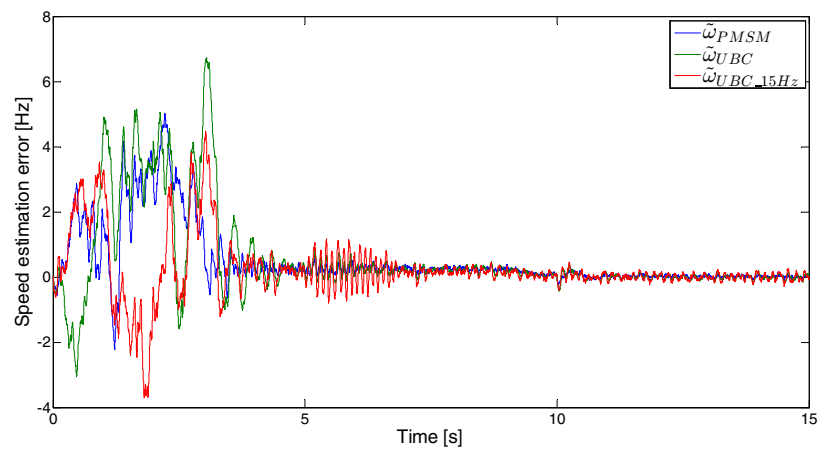

Fig. 6. Unbalance compensation response with different speed estimation signals as input.

Despite the low speed control bandwidth selected for the sensorless vector control, the bandwidth of the drive's estimated speed is still relatively large and results in a large current ripple. Hence, the speed control loop with

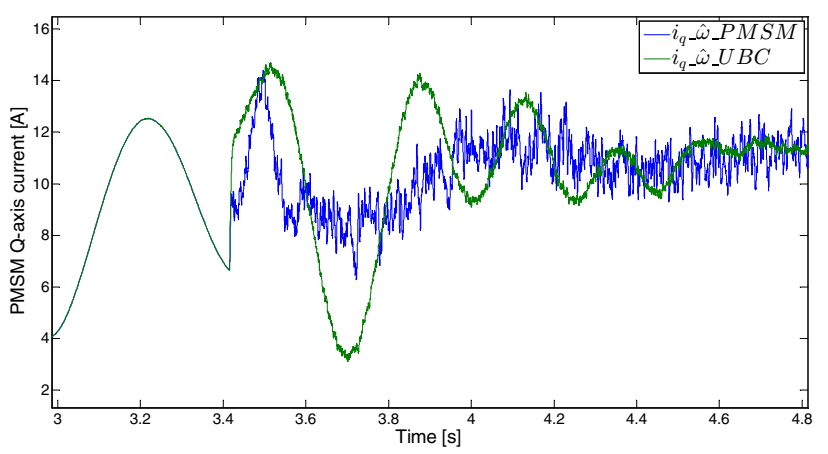

Fig. 7. Speed control response with unbalance compensator's speed estimate in the speed control feedback.

the unbalance compensation's estimated speed used as the speed feedback in the vector control is considered as shown in Fig. (7). It can be seen that the resulting current ripple caused by noise in the estimated speed signals have been reduced significantly. The speed estimation bandwidth from the unbalance compensator is dependent on the operating frequency, hence the large transient when switching over at low speed.

\section{CONCLUSIONS AND FUTURE WORK}

A step towards using the unbalance compensation speed estimation signal in the vector control has been presented, by using the unbalance control estimated speed in the speed control loop of the vector controller. For this application which has a large rotor inertia, the speed estimation bandwidth of the unbalance control loop was large enough to be used in the vector control. A modified start-up procedure which has been customized for use in AMB systems (which have very low friction and hence very low speed perturbation damping) have been presented. The modified start-up procedure can also be used in sensorless drive schemes which do not utilize AMBs.

Future work include converting the unbalance control presented by Lee et al. [2002] to operate in COG coordinates, to investigate if it can compensate the unbalance of highly gyroscopic rotors. Currently the unbalance compensation speed estimate is not useful for redundancy, because a phase-lock mechanism still need to be developed such that the estimated angular position of the unbalance control can be locked onto the absolute phase of the rotor if it is to be used in a vector control scheme as previously suggested by Lee et al. [2002]. The modified unbalance control in cooperation with the PMSM drive control on the physical flywheel energy storage system need to be implemented for validation.

\section{REFERENCES}

Alain, S., Lechable, B., 1996. An overview on Aerospatiale magnetic bearing products for spacecraft attitude control and for industry. In: Third International Symposium on Magnetic Suspension Technology. pp. 217-226.

Batzel, T. D., 2000. Electric propulsion using the permanent magnet synchronous motor without rotor position transducers. Ph.D. thesis, The Pennsylvania State University. 
Bleuler, H., Gahler, C., Herzog, R., Larsonneur, R., Mizuno, T., Siegwart, R., 1994. Application of digital signal processors for industrial magnetic bearings. IEEE Transactions on Control Systems Technology 2 (4), 280 289.

Burdet, L., 2006. Active magnetic bearing design and characterization for high temperature applications. Ph.D. thesis, EPFL.

Dimond, T., Allaire, P., Mushi, S., Lin, Z., Yoon, S. Y., 2012. Modal tilt/translate control and stability of a rigid rotor with gyroscopics on active magnetic bearings. International Journal of Rotating Machinery 2012, 1-10.

Gerlach, B., Ehinger, M., Seiler, R., 2006. Low noise five-axis magnetic bearing reaction wheel. Mechatronic Systems 4 (1), 572-577.

Gouws, R., van Schoor, G., 2009. Multiple frequency fault detection, correction and identification of vibration forces on the rotor of a rotational active magnetic bearing system. SAIEE Africa Research Journal 99 (December), 114-123.

Grochmal, T., Lynch, A., 2007. Precision tracking of a rotating shaft with magnetic bearings by nonlinear decoupled disturbance observers. IEEE Transactions on Control Systems Technology 15 (6), 1112-1121.

Herzog, R., Bühler, P., Gähler, C., Larsonneur, R., 1996. Unbalance compensation using generalized notch filters in the multivariable feedback of magnetic bearings. IEEE Transactions on Control Systems Technology 4 (5), 580-586.

Janse van Rensburg, J. J., 2007. Development of a flywheel energy storage system - uninterrupted power supply (FLY-UPS). Master's thesis, North-West University.

Jastrzebski, R., Pöllänen, R., Pyrhönen, O., Kärkkäinen, A., Sopanen, J., 2006. Modeling and implementation of active magnetic bearing rotor system for FPGA-based control. In: 10th International Symposium on Magnetic Bearings.

Kejian, J., Changsheng, Z., Ming, T., 2012. A uniform control method for imbalance compensation and automation balancing in active magnetic bearing-rotor systems. Journal of Dynamic Systems, Measurement, and Control 134 (2), 021006.

Kim, J.-S., Sul, S.-K., 2000. New approach for highperformance PMSM drives without rotational position sensors. IEEE Transactions on Power Electronics 12 (5), 904-911.

Kruger, G. L., Grobler, A. J., Holm, S. R., 2013. Improved non-ideality compensation for the $\mathrm{V} / \mathrm{f}$ controlled permanent magnet synchronous motor. In: IEEE International Conference on Industrial Technology (ICIT). pp. 331-336 .

Kshirsagar, P., Burgos, R. P., Lidozzi, A., Jang, J., Wang, F., Boroyevich, D., Sul, S.-K., 2006. Implementation and sensorless vector-control design and tuning strategy for SMPM machines in fan-type applications. In: Conference Record of the 2006 IEEE Industry Applications Conference. 41st IAS Annual Meeting. Vol. 4. IEEE, pp. 2062-2069.

Lee, W.-L., Schumacher, W., Canders, W.-R., 2002. Unbalance compensation on AMB system without a rotational sensor. In: 8th International Symposium on Magnetic Bearings. Vol. 46. pp. 211-216.
Lees, A., Sinha, J., Friswell, M., Aug. 2009. Model-based identification of rotating machines. Mechanical Systems and Signal Processing 23 (6), 1884-1893.

Lima, F., Rosa, F. C., Fumagalli, M. A., 2010. Simulation of a neurofuzzy high speed estimation applied to magnetic bearing systems. In: 12th International Symposium on Magnetic Bearings. pp. 27-33.

Myburgh, S., 2007. The development of a fully suspended AMB system for a high-speed flywheel application. Master's thesis, The School of Electrical, Electronic and Computer Engineering, North-West University.

Perera, P. D. C., Blaabjerg, F., Pedersen, J. K., Thogersen, P., 2003. A sensorless, stable $\mathrm{V} / \mathrm{f}$ control method for permanent-magnet synchronous motor drives. IEEE Transactions on Industry Applications 39 (3), 783-791.

Polajžer, B. (Ed.), 2010. Magnetic bearings, theory and applications. Sciyo.

Sawicki, J., Friswell, M., Baaklini, G., 2008. Rotor crack detection using induced combination resonances. In: 4th European Workshop on Structural Health Monitoring. pp. $142-149$.

Schweitzer, G., Maslen, E. H., Bleuler, H., Cole, M., Keogh, P., Larsonneur, R., Nordman, R., Okada, Y., Traxler, A., 2009. Magnetic bearings: theory, design, and application to rotating machinery.

Worlitz, F., Jun. 2011. Active magnetic bearings - A product of mechatronics. In: 10th International Workshop on Electronics, Control, Measurement and Signals. pp. 1-3.

\section{Appendix A. NUMERICAL VALUES OF MODEL PARAMETERS}

The parameters used for the AMB simulation are presented in Table A.1.

Table A.1. AMB and rotor parameters.

\begin{tabular}{|l|c|c|}
\hline Parameter description & Symbol & Value \\
\hline \hline AMB current force factor & $k_{i}$ & $60.5 \mathrm{NA}^{-1}$ \\
\hline AMB disp. force factor & $k_{s}$ & $303 \times 10^{3} \mathrm{Nm}^{-1}$ \\
\hline Rotor weight & $M$ & $18.3 \mathrm{~kg}$ \\
\hline Bearing disp. from COG & {$[a, b]$} & {$[-164,64.4] \times 10^{-3} \mathrm{~m}$} \\
\hline Sensor disp. from COG & {$[c, d]$} & {$[-190,95.4] \times 10^{-3} \mathrm{~m}$} \\
\hline Transverse MOI & $J_{t}$ & $0.11575 \mathrm{~kg} \cdot \mathrm{m}^{2}$ \\
\hline Air-gap & $g_{0}$ & $500 \times 10^{-6} \mathrm{~m}$ \\
\hline
\end{tabular}

The parameters of the PMSM used in the simulation are presented in Table A.2. The electrical parameters are from measurements, the rotor inertia from the rotor design Janse van Rensburg [2007] and the mechanical friction parameters are estimated.

Table A.2. PMSM parameters.

\begin{tabular}{|l|c|c|}
\hline Parameter description & Symbol & Value \\
\hline \hline \#Pole pairs & $z_{p}$ & 1 \\
\hline Stator inductance & $L_{d}$ & $500 \times 10^{-6} \mathrm{H}$ \\
\hline Stator resistance & $r_{s}$ & $0.172 \Omega$ \\
\hline PM flux linkage & $\lambda_{p}$ & $46.4 \times 10^{-3}$ Wb.t \\
\hline Rotor polar MOI & $J_{p}$ & $0.107 \mathrm{~kg} . \mathrm{m}^{2}$ \\
\hline Viscous friction coefficient & $B$ & $1 \times 10^{-6}$ N.m.s.rad \\
\hline Coulomb friction torque & $T_{l}$ & $5 \times 10^{-6}$ N.m \\
\hline
\end{tabular}

